This paper has been accepted for publication in Developmental Science. The final article will be available, upon publication, via its DOI:10.1111/desc.13187

\title{
Parietal and hippocampal hyper-connectivity is associated with low math achievement in adolescence - a preliminary study
}

\author{
Roberto A. Abreu-Mendoza ${ }^{1}$, Melanie Pincus ${ }^{1}$, Yaira Chamorro ${ }^{2}$, Dietsje Jolles ${ }^{3}$, Esmeralda \\ Matute $^{2}$, and Miriam Rosenberg-Lee ${ }^{1,4}$ \\ ${ }^{1}$ Department of Psychology, Rutgers University - Newark \\ ${ }^{2}$ Instituto de Neurociencias, CUCBA, Universidad de Guadalajara, Guadalajara, Jalisco, México \\ ${ }^{3}$ Department of Education and Child Studies, Leiden University, Leiden, The Netherlands \\ ${ }^{4}$ Center for Molecular and Behavioral Neuroscience, Rutgers University-Newark
}

\section{Author's Note}

The authors declare that there is no conflict of interest. The data that support the findings of this study are available from the corresponding author, upon reasonable request. The authors wish to thank Daniel Zarabozo-Hurtado for his assistance in data collection, Diana Ávalos and Daniel Romero for their help in evaluation and data collection, and Hillary Contreras for their assistance in database collection. We also thank all participants and their families, and the high schools, principals, and teachers who assisted with this project. This study was funded by a grant from Consejo Nacional de Ciencia y Tecnología (CONACyT): Investigación en Fronteras de la Ciencia Grant No. 783. Correspondence concerning this article should be addressed to Roberto A. Abreu-Mendoza, roberto.abreu@rutgers.edu, or Esmeralda Matute, ematute@redudg.udg.mx. 


\section{Research Highlights}

- In childhood, low math is associated with hyper-connectivity of the intraparietal sulcus (IPS).

It is unknown whether hyper-connectivity is also found in poor-performing adolescents.

- IPS functional connectivity in adolescents was negatively associated with math ability, as in children. However, the temporooccipital target regions resembled areas implicated in adult studies.

- Connectivity of the hippocampus was negatively related to math ability, contrary to studies predicting mathematical learning from hippocampal connectivity.

- Our findings suggest that hyper-connectivity of IPS and hippocampus, areas important for math cognition, remains a hallmark of low math ability into adolescence. 


\begin{abstract}
Mathematical cognition requires coordinated activity across multiple brain regions, leading to the emergence of resting-state functional connectivity as a method for studying the neural basis of differences in mathematical achievement. Hyper-connectivity of the intraparietal sulcus (IPS), a key locus of mathematical and numerical processing, has been associated with poor mathematical skills in childhood, whereas greater connectivity has been related to better performance in adulthood. No studies to date have considered its role in adolescence. Further, hippocampal connectivity can predict mathematical learning, yet no studies have considered its contributions to contemporaneous measures of math achievement. Here, we used seed-based resting-state fMRI analyses to examine IPS and hippocampal intrinsic functional connectivity relations to math achievement in a group of 31 adolescents (mean age $=16.42$ years, range 15-17), whose math performance spanned the $1 \%$ to $99 \%$ percentile. After controlling for IQ, IPS connectivity was negatively related to math achievement, akin to findings in children. However, the specific temporooccipital regions were more akin to the posterior loci implicated in adults. Hippocampal connectivity with frontal regions was also negatively correlated with concurrent math measures, which contrasts with results from learning studies. Finally, hyper-connectivity was not a global feature of low math performance, as math performance did not modulate connectivity of Heschl's gyrus, a control seed not involved in math cognition. Our results provide preliminary evidence that adolescence is a transitional stage in which patterns found in childhood and adulthood can be observed; most notably, hyperconnectivity continues to be related to low math ability into this period. Keywords: Functional connectivity, Hippocampus, Individual differences, Intraparietal sulcus, Math achievement.
\end{abstract}




\section{Parietal and hippocampal hyper-connectivity is associated with low math achievement in adolescence - a preliminary study}

In modern society, mathematical achievement has impacts beyond the classroom, affecting life outcomes, from employability (Handel, 2016) and attained socioeconomic status (Ritchie \& Bates, 2013), to financial (Gerardi et al., 2013) and health (Reyna et al., 2009) outcomes. Given the wide-ranging repercussions of poor math ability, it becomes crucial to understand the neurobiological underpinnings of individual differences in mathematical attainment. The advent of functional neuroimaging held the promise of identifying reliable hallmarks of individual differences in mathematical ability; yet, no clear-cut pattern has emerged from task-based functional MRI (fMRI) studies. In childhood, there have been reports of both lower (Haist et al., 2015; Kovas et al., 2009; Price et al., 2007) and higher activity (Iuculano et al., 2015; Kovas et al., 2009; McCaskey et al., 2017; Rosenberg-Lee et al., 2015), depending on the level of task difficulty, the age of the participants, and the brain region examined. Yet, the cumulative nature of math knowledge complicates attempts to examine a single mathematic skill with the same task design across developmental stages and ability levels.

To overcome the limitations of task-based fMRI paradigms, researchers have taken advantage of progress in resting-state fMRI methods over the last decade. Resting-state fMRI employs a simple instruction for participants (e.g., remain still while fixating on a cross-hair or with your eyes closed) while collecting spontaneous fluctuations in brain activity and computing temporal dependency between these spatially remote variations in activity (Fox \& Raichle, 2007; Van Den Heuvel \& Pol, 2010). By studying the connectivity patterns between spontaneous fluctuations in activity across different regions of the brain, resting-state fMRI analyses move away from associating skills with single areas toward a network approach (Cole et al., 2014). 
This stance is especially apt for studying complex skills such as mathematical cognition, which require coordinated activity across multiple brain regions (Dehaene \& Cohen, 2007; Menon, 2016).

Task-based studies have highlighted the critical role of the intraparietal sulcus (IPS) in numerical magnitude processing (Arsalidou \& Taylor, 2011; Sokolowski et al., 2017) and in distinguishing children with typical performance from those with low math ability (Price et al., 2007; Rosenberg-Lee et al., 2015). Moreover, recent work has begun to highlight the importance of the hippocampus, involved in encoding, consolidation, and retrieval of numerical facts (Cho et al., 2011; Qin et al., 2014; Rosenberg-Lee et al., 2018). Beyond these core regions, other work has also identified brain regions that support abilities related to math cognition, such as the lateral occipital cortex, involved in decoding visual numerical symbols, the insula, related to attention, the dorso- and ventrolateral prefrontal cortex, associated with executive functions (Kaufman et al., 2011; Kucian, 2016; Menon, 2016). In particular, resting-state fMRI offers a means to examine how connectivity patterns support math skills, which can be employed across individuals with different math levels and at different developmental stages due to its task-free nature.

\section{Resting-state IPS connectivity and math ability}

The first paper to investigate patterns of resting-state functional connectivity associated with low math ability was Jolles, Ashkenazi, et al. (2016). This study examined differences in the functional connectivity of the intraparietal sulcus of children with mathematical disabilities (MD) and typically developing children. Contrary to the idea that greater functional connectivity reflects greater neural efficiency, children with MD showed higher IPS functional connectivity, relative to their typically developing peers, with frontoparietal regions, including the superior 
frontal gyrus, superior parietal lobule, and supramarginal gyrus. Coupled with findings of overactivity during task (Iuculano et al., 2015; Rosenberg-Lee et al., 2015), the authors interpreted this hyper-connectivity in children with MD as reflecting difficulty in appropriately modulating connectivity between regions, across tasks and contexts.

Importantly, this negative association has recently been replicated by another research group (Price et al., 2018). They employed five parietal seeds - the three cytoarchitectonic subdivisions of the IPS and those two of the angular gyrus (Choi et al., 2006; Scheperjans et al., 2008) - to examine the relationship between parietal functional connectivity in first grade and arithmetic ability in second grade. Consistently with Jolles, Ashkenazi, et al. (2016), the wholebrain analyses indicated that children with low math ability showed higher functional connectivity of two of the left IPS subdivisions (hIP1 and hIP2) with the right parahippocampal gyrus and the left middle temporal gyrus, respectively, and the left posterior portion angular gyrus with the left superior frontal gyrus, even after controlling for reading, spatial attention, and working memory. However, they also found that inter-hemisphere functional connectivity of the IPS showed a positive relationship with math skills. On balance, these studies suggest the IPS hyper-connectivity as a candidate for a neural signature of low math ability in childhood.

Studies with adult populations reveal a more complex picture (Koyama et al., 2017, 2020; Skagerlund et al., 2018). When examining the functional connectivity of the IPS and AG subdivisions in adults, Skagerlund et al. (2018) found that connectivity of the IPS (subdivision hIP1) with the superior frontal gyrus was negatively correlated with arithmetic fluency. However, after controlling for the contributions of inhibition, shifting, and working memory skills, this relationship was no longer observed. Instead, functional connectivity of IPS subdivisions hIP1 and hIP3 with the premotor cortex and supramarginal gyrus showed a positive 
relationship, even after controlling for executive function skills. Beyond the parietal lobe, Koyama et al. (2017) found that connectivity of the middle frontal gyrus with the insula and anterior cingulate cortex was positively related to math performance, while connectivity of the this same region with parietotemporal regions and the ventromedial prefrontal cortex was negatively related. These studies suggest that in adulthood, IPS functional connectivity has the opposite pattern of childhood, namely, worse math skills are associated with lower connectivity of the IPS.

Adolescence has often been overlooked in research of math cognition, not only in behavioral studies (Schneider et al., 2017) but also in neuroimaging research. An outstanding question is whether the neural signature of low math ability in adolescence, at rest, resembles the over-connectivity observed during childhood or the under-connectivity found in adulthood, or some intermediary position. Only two studies to date have examined functional connectivity in adolescents, both in task-based designs. In Abreu-Mendoza et al. (2020), functional connectivity among areas activated in a non-symbolic comparison task was negatively associated with math performance in adolescents aged 15 to 17 . In particular, adolescents with low ability showed higher connectivity in a set of posterior regions: the lateral occipital cortex, the cuneal cortex, the lingual gyrus, and the precuneus. In contrast, in a longitudinal study, Battista et al. (2018) found a positive relationship between IPS connectivity and individual differences in math ability in the transition from childhood to early adolescence. Using the left hIP3 as the seed, the authors report that during an arithmetic task, children's IPS connectivity with frontal areas decreased over time, from ages 7 to 14, while connectivity with parietotemporal regions strengthened during this period. Critically, regardless of time point, inter-hemispheric IPS connectivity was positively 
related to individual differences in math ability: children with high math ability consistently showed high connectivity between the left and right IPS over the six-year span.

In summary, although these task-based studies report divergent evidence on the direction of the relationship between functional connectivity and math ability, they both suggest a developmental shift, from anterior to posterior regions, in the functional connections relevant for math ability. Yet, this shift could reflect age-related changes that are specific to the tasks used in those studies. Critically, no study to date has examined the resting-state functional connectivity and its relation to math in adolescence. Here, we aimed to investigate, for the first time, the relationship between IPS resting-state functional connectivity and math ability in adolescents aged 15 to 17 .

\section{Math learning and hippocampus}

Beyond the IPS, growing evidence points to the hippocampus as brain region that plays a critical role in numerical cognition, particularly for its role in math fact learning (Qin et al., 2014; Supekar et al., 2013). Recently, the well-established role of the hippocampus in declarative memory has been incorporated into theories of the neural basis of math cognition (Kucian, 2016; Menon, 2016). Specifically, the hippocampus, together with regions of the prefrontal cortex, participates in the formation and retrieval of long-term memories and maturation of memorybased strategies (Klein et al., 2013; Menon, 2016). For example, both longitudinally (Qin et al., 2014) and following intensive tutoring (Rosenberg-Lee et al., 2018), there is an increase in connectivity between the hippocampus and frontal or parietal cortex, which in turn correlates with behavioral improvements in fact memorization.

Intrinsic connectivity of the hippocampus is also predictive of learning gains from math interventions in typically developing children. Supekar et al. (2013) found that children who 
showed higher resting-state connectivity of the right hippocampus with temporal and frontal areas were those who benefit the most from an eight-week intervention that targeted arithmetic fluency skills. In a later study, Jolles, Supekar, et al. (2016) reported that children who underwent this same intervention showed plasticity of the IPS connectivity with frontal and temporooccipital regions, including the bilateral hippocampus. More specifically, changes in efficiency in solving arithmetic problems were positively correlated with changes in the functional connectivity of these areas.

Thus far, studies of task-based and resting state connectivity have only considered the hippocampus' role in predicting learning gains in math ability. No studies have examined the relationship of hippocampal connectivity with contemporaneously measure math ability, which leaves an incomplete characterization of the contributions of this region to math skills. For instance, as strong hippocampal functional connectivity predicts students' future success in learning mathematical content (Supekar et al., 2013), we could infer that individuals with currently high math ability are likely apt learners and would show high connectivity. Under this scenario, we expect a positive relationship between higher connectivity of the hippocampus and math ability.

Alternatively, if a hallmark of low math ability is a hyper-connectivity of brain areas involved numerical cognition, then the hippocampal functional connectivity should show a negative correlation with math skills, as found in studies with IPS seeds (Jolles, Ashkenazi, et al., 2016; Price et al., 2018). Indirect evidence seems to support this scenario. Children with MD show higher task-based IPS connectivity with the right hippocampus in comparison to typically developing children (Michels et al., 2018). A third possibility is that individual differences in current math ability might only be reflected by the IPS functional connectivity, as the IPS is a 
crucial hub in mathematical cognition (Arsalidou \& Taylor, 2011; Sokolowski et al., 2017).

Under this view, we would not expect a relationship of general math skills with connectivity of any other seed but the IPS. To adjudicate between these alternatives, we examined the functional connectivity of adolescents using the hippocampus as a seed.

\section{IQ and math ability}

The moderate but highly consistent relationship between academic skills and general intelligence (Peng et al., 2019) pose a challenge for examining individual differences in math skills. Typically, to study the neural underpinning of math ability, researchers have contrasted two groups: individuals with MD vs. individuals with typical performance (Iuculano et al., 2015; Jolles, Ashkenazi, et al., 2016; Michels et al., 2018; Price et al., 2007; Rosenberg-Lee et al., 2015). Matching these groups on non-mathematical cognitive skills enables isolating the brain regions associated with math ability from those related to other cognitive factors, (e.g., IQ and working memory). However, a pitfall of this matching approach is that it can lead to nonrepresentative groups (Dennis et al., 2009). Moreover, this approach typically excludes individuals in the upper end of the math ability range, who can be difficult to match in cognitive capacity. In fact, a recent systematic review of research on neural and cognitive correlates of math giftedness reported that there is no study that has explored the functional connectivity of individual with high math skills (Myers et al., 2017). As an alternative to matching group-wise, recent studies have opted to use math ability as a continuous variable and include IQ as a covariate (Price et al., 2018; Skagerlund et al., 2018). In the current study, we consider math achievement after accounting for IQ, an approach that enables us to include participants with math scores ranging from the $1 \%$ to $99 \%$ percentile (Abreu-Mendoza et al., 2019). 
However, the behavioral correlation between IQ and math ability raises the possibility of overlap between regions that are part of the math cognition networks (Fias et al., 2013) and those related to IQ (Dubois et al., 2018). In fact, theories of the neural correlates of intelligence have proposed areas of the frontoparietal and the default mode networks as critical to explaining $g$ factor (Dubois et al., 2018; Jung \& Haier, 2007), regions also implicated in math cognition. Recently, Koyama et al. (2020) examined possible common areas that contribute to both IQ and academic skills (reading and math ability) and found that the regional homogeneity, a measure of local functional connectivity, of the thalamus was negatively related to both reading and math as well as IQ. However, this result still leaves unanswered whether the IPS connectivity reported in previous studies is specifically related to math skills or overlaps with that of general ability. To address the specificity of our results relating math ability, after accounting for IQ, to functional connectivity of the IPS and hippocampus, we also investigate the independent functional connectivity of IQ.

\section{The current study}

The first aim of this study was to investigate whether the IPS functional connectivity related to math achievement in adolescents was consistent with the direction reported in children (higher connectivity, worse math performance) or adults (lower connectivity, worse math performance). For consistency with prior studies, we first examined the relationship between IPS functional connectivity and raw math achievement scores in a group of adolescents with a wide range of math ability. Then, given the robust correlation between math achievement and IQ, we followed up these analyses by interrogating whether IPS connectivity was related to math ability after accounting for IQ. The second aim was to probe whether the relationship between the hippocampal intrinsic connectivity and current math achievement, was akin to positive 
relationship found between hippocampal connectivity and math learning gains. To achieve this goal, we also considered both the relationship of hippocampal functional connectivity with raw achievement and after controlling for IQ.

To preview, we found negative associations between math achievement and connectivity of both the IPS and hippocampus. Notably, these effects were strongest when we controlled for IQ. Thus, our final aim was to investigate the cognitive and anatomical specificity of these results. To determine whether IPS and hippocampal hyper-connectivity was specific to low-math skills or was associated with low general cognitive ability, we examined the relationship between the connectivity of these areas and IQ. To assess whether hyper-connectivity was specific to these critical regions of math cognition, we examined connectivity of an area not associated with math cognition, Heschl's gyrus. We reasoned that if relations between math ability and functional connectivity are restricted to regions involved in math cognition (i.e., IPS and hippocampus), we should not observe any association with Heschl's gyrus connectivity. However, if hyperconnectivity is a global feature of poor math ability, we might expect to find it present in Heschl's gyrus connectivity, as well.

\section{Methods}

\section{Participants}

Participants were part of a large-scale research project that aimed to obtain normative scores for the mathematical abilities in Mexican adolescents (Abreu-Mendoza et al., 2019). A subsample of these students completed both an fMRI task run (reported elsewhere, AbreuMendoza et al., 2020) and the resting state run reported here. Adolescents from three public high schools in Guadalajara, Mexico, participated in the normative study. For the imaging portion of the study, they were recruited based on their performance on the Math Computation subtest of 
the Wide Range Achievement Test-4, WRAT-4 (Wilkinson \& Robertson, 2006), which was translated to Spanish language, including mathematical notation conventions used in Mexico (Abreu-Mendoza et al., 2019). Participants were adolescents whose ages ranged from 15 to 17 years, which corresponds to the ages of high-school students in Mexico (secondary school encompasses three years, equivalent to grades 10 to 12 years in the US system).

Fifty-one adolescents were selected to form three mathematical achievement groups: mathematical disability (MD), typical performance, and mathematical talent. MD was defined as having a score at least $1.5 \mathrm{SD}$ below the mean on the WRAT Math Computation subtest, according to the norms for Mexican high school students (Abreu-Mendoza et al., 2019), and a score below the 10th percentile on the Written Math subtest of the Evaluación Neuropsicológica Infantil (ENI, Matute et al., 2007). Typical performance was considered as having a score within the range of $\pm 1.5 \mathrm{SD}$ from the Mexican mean on the WRAT Math Computation and a score above the 10th percentile on the ENI. Mathematical talent was defined as a score of $\geq 1.5 \mathrm{SD}$ from the Mexican mean and a score above the 90th percentile on the ENI Written Math subtest. Participants from all groups were also required to 1) have an IQ $\geq 80$ on a short form of the Mexican adaptation of the WISC-IV (Wechsler, 2007), which included Vocabulary and Matrix Reasoning subtests, 2) have a reading speed score above the $10^{\text {th }}$ percentile on the Reading a Text Aloud subtest from the ENI (Matute et al., 2007), 3) be free of any fMRI contraindications (e.g., metallic implants, orthodontic braces, or claustrophobia), and 4) not meet the diagnostic criteria for attention deficit hyperactivity disorder (ADHD), based on a parent questionnaire that probes DSM-5 criteria (American Psychiatric Association, 2013).

Of the 51 neuroimaging participants, eleven adolescents were excluded due to excessive head motion, defined as an average framewise displacement (FD) larger than $0.5 \mathrm{~mm}$ and more 
than $40 \%$ of the volumes censored. Every structural image was reviewed by a neuroradiologist and seven participants were excluded due to incidental findings (e.g., cysts and adenomas).

Finally, two participants asked to end the session prior to collection of the resting-state scan. The final sample included 31 adolescents between the ages of 14.97 to 17.83 (mean age $=16.42$, SD $=0.80,16$ females and 15 males). Due to the small number of participants in each group (Mathematical disability $=7$, Typical performance $=11$, and Mathematical talent $=13)$, math performance was examined as a continuous (i.e., number of correct responses in WRAT Math Computation) rather than a categorical variable.

The descriptive statistics of age, cognitive and academic achievement measures, and the coefficients for the correlations among these measures are reported in Table 1. WRAT computation scores ranged from 10 to 38 , which corresponds to $<1 \%$ to $99 \%$ percentile based on a larger norming study of Mexican adolescents (Abreu-Mendoza et al., 2019). Although we did not have access to standard WRAT age norms, age was not correlated to the WRAT Math Computations subtest $(r(29)=-.04, p=.814)$. In contrast, IQ was strongly related to the number of correct responses in the WRAT subtest $(r(29)=.72, p<.001$, Figure 1A). Thus, to control for the effect of IQ in subsequent imaging analyses, we performed a linear regression analysis with the z-transformed IQ scores as the predictor and z-transformed WRAT scores as the dependent variable to generate the standardized Math-IQ residuals. Notably, although WRAT z-scores were not normally distributed (differed significantly from normal according to a Shapiro-Wilk test, W $=.90, p=.006$, Figure $1 \mathrm{~B})$, Math-IQ residuals were normally distributed $(\mathrm{W}=.95, p=.210$, Figure 1C). Age was also not correlated with Math-IQ residuals $(r=.05, p=.801)$.

All participants and their parents gave written informed consent and received a travel reimbursement of approximately USD 15 at the end of the MRI session. All protocols were 
approved by the ethics committee of the Institute of Neurosciences of the Universidad de Guadalajara.

\section{Standardized cognitive assessments}

\section{Mathematical ability}

WRAT Math Computation. This timed subtest from the WRAT-IV (Wilkinson \& Robertson, 2006) subtest comprises 40 items in which participants have to solve written mathematical problems of increasing difficulty, with a 15-min time limit. It begins with a simple addition problem and ends with the reduction of a rational expression to its lowest terms.

ENI Written Math subtest. In this subtest of the standardized ENI (Matute et al., 2007), participants had a maximum of 10 minutes to answer 14 problems of increasing difficulty. The simplest items involved single-digit arithmetic, whereas the most difficult items involved the addition of like fractions (e.g., $\frac{3}{5}+1 \frac{1}{5}+\frac{1}{5}$ ) and solving a simple linear equation (e.g., $4 \mathrm{x}+2=$ $10)$.

WISC Arithmetic subtest. This subtest of the WISC-IV (Wechsler, 2007) was used to assess the ability to solve mathematical problems presented orally. For children older than 10, this test comprises 28 problems of increasing difficulty in which they have 30 seconds to solve each problem mentally. The simplest problem for this age range involves a problem that involve computing a single-digit arithmetic operation, while the most two difficult items comprise algebraic problems embedded in a short text.

\section{Reading ability}

ENI Reading a Text Aloud. In this subtest, participants are asked to read aloud a 101word text, Tontolobo y el Carnero ("The Silly Wolf and the Ram"); then, they are then asked four text-comprehension questions. 


\section{$I Q$}

WISC Short Form. IQ was estimated using a short form of the WISC-IV (Wechsler, 2007), which included the Vocabulary and Matrix Reasoning subtests. According to Sattler (2010), this form is one of the 10 best subtest combinations for IQ estimation and has high reliability (.93) and validity (.87) scores.

\section{MRI procedure}

The MRI protocol was composed of an initial 1-minute T2-weighted brain volume, followed by an 8-minute high-resolution T1-weighted brain volume, a 26-minute fMRI task, a 6minute DTI sequence. The session ended with a 10-minute resting-state sequence. During this last sequence, participants saw a white fixation cross at the center of a black screen and were instructed to 'Look at the fixation cross and try not to think of anything in particular.' Taskbased imaging results are reported elsewhere (Abreu-Mendoza et al., 2020). A neuroradiologist inspected the anatomical images to rule out the presence of neuro-pathologies. Participants who showed incidental findings were excluded from subsequent analyses.

\section{MRI data acquisition}

Data were collected using a 3T scanner (Philips Ingenia; Best, The Netherlands) with an 8-channel head coil. The resting-state scan was comprised of 300 contiguous functional volumes using an echo-planar imaging (EPI) sequence $(\mathrm{TR}=2,000 \mathrm{~ms}$, TE $30 \mathrm{~ms}, \mathrm{FOV}=230 \mathrm{x} 230 \mathrm{x}$ $116 \mathrm{~mm}$; matrix $=96 \times 94 ; 29$ slices per volume, thickness $=4 \mathrm{~mm}$ with no gap, voxel size $=$ $2.40 \times 2.44 \times 4 \mathrm{~mm})$. The T1-weighted brain volume was acquired using a high-resolution 3D Turbo Field Echo $(\mathrm{TFE})$ sequence $(\mathrm{TR}=4900 \mathrm{~ms}, \mathrm{TE}=210 \mathrm{~ms}, \mathrm{FOV}=240 \times 240,170$ slices, thickness $=1 \mathrm{~mm}$ with no gap, voxel size $=1 \mathrm{~mm}$ isotropic).

\section{Movement}


Head movement was measured using FSL Motion Outliers with FD as defined in Power et al. (2012). Among participants meeting the criteria of less than $0.5 \mathrm{~mm}$ FD and less $40 \%$ of the volumes censored (i.e. at least 6 minutes of resting state time, Van Dijk et al., 2010), mean FD was $0.24 \mathrm{~mm}(\mathrm{SD}=0.08$, range $=0.13-0.47)$. Volumes that exceeded FD of $0.3 \mathrm{~mm}$ were censored (mean percentage of censored volumes $=19.24, \mathrm{SD}=11.96$, range $=0.66-40 \%)$. No significant correlations between number of censored volumes and WRAT-Math Computation scores or Math-IQ residuals were observed $(p s>.180)$.

\section{fMRI data preprocessing}

Preprocessing was conducted using an in-house pipeline, employing FSL (FMRIB Software Library) version 6.00 and AFNI version 19.3.11(Cox, 1996). As a first step, images were resampled to a resolution of $2 \mathrm{~mm}$. AFNI's afniproc.py was implemented to perform motion correction, which consisted of registering EPI images with the individual's anatomical images, which in turn were registered to standard space (MNI). FMRIB's Automated Segmentation Tool (FAST, Y. Zhang et al., 2001) was used to segment white matter and cerebrospinal fluid form each participant's structural image. Then, AFNI's 3dmaskave was used to extract the average time courses within each mask. Next, AFNI's 3dDeconvolve was used to perform a nuisance regression with the six standard motion parameters, white matter, and ventricle signals. Finally, spatial smoothing using a Gaussian kernel (6 mm full-width at halfmaximum) and a bandpass filter $(0.009$ to $0.080 \mathrm{~Hz})$ to remove high-frequency artifacts were performed using AFNI's 3dmerge and 3dBandpass, respectively.

\section{Functional connectivity analysis}

\section{Selection of regions of interest}


Two anatomical regions of interest (ROI) were selected, the IPS and the hippocampus. To define the former, we combined the three IPS subdivisions from the Juelich histological atlas, 25\% threshold (Choi et al., 2006; Scheperjans et al., 2008), while the hippocampus was defined using the Anatomical Automatic Labeling (AAL) atlas (Rolls et al., 2020). Following Jolles, Supekar et al. (2016), we chose the Heschl gyrus as our anatomical region of control, which was also defined using the AAL atlas. In total, there were six ROIs: three regions, across two hemispheres (Figure 2).

\section{Whole-brain functional connectivity analysis}

For each participant, we first extracted the time series from the six ROIs. Then, we performed general linear models (GLMs) using AFNI's 3dDeconvolve with the motion-corrected images. As nuisance regressors for motion were already incorporated in the preprocessing steps, each GLM only included the time course of one of the ROIs as the predictor and a column indicating which volumes would be censored due to excessive movement, defined as an FD larger than $0.3 \mathrm{~mm}$. Group-level analyses were performed using a one-sample $t$-test, using the modified AFNI's 3dttest++, for each functional connectivity map of each ROI with either one of three covariates: number of correct responses in the WRAT Math computation subtest, the MathIQ residuals (see Neuropsychological profile section), and IQ scores. Due to the recent concerns about the potential over-estimation of Type I control of both the former version of $3 \mathrm{dClustsim}$ and its precursor AlphaSim (Eklund et al., 2016), we employed AFNI's 3dttest++ with the Clustsim option, which uses $t$-test randomization to minimize the false positive rate (Cox et al., 2017). For these contrasts maps, we applied the whole brain mask and used a height threshold of $p<.01$, with family-wise error (FWE) corrections for multiple comparisons at the cluster level $(p$ $<.10)$. The specific cluster size and cluster extend $p$-value for each analysis are reported in the 
Results section. Cluster extent thresholds were estimated with 10,000 iterations of a Monte Carlo simulation using Clustsim; the resulting cluster extent size is reported for each analysis.

To facilitate comparison of our results with those presented in Jolles, Ashkenazi et al. (2016), we report, as supplementary analyses, IPS connectivity analyses with the same threshold and the same grey matter mask as Jolles Ashkenazi et al. (2016). Specifically, we applied a grey brain mask and then employed a height threshold of $p<.01$, with clusters exceed spatial extent 128 voxels. This cluster size corresponds to family-wise error (FWE) corrections for multiple comparisons at the cluster level of $p<.01$, reported by Rosenberg-Lee, Barth, \& Menon (2011) using a Monte Carlo method similar to the one used by the former AFNI's AlphaSim program (Forman et al., 1995; Ward, 2000).

Anatomical labels were defined by manually entering MNI peak coordinates into the Harvard-Oxford subcortical and cortical structural probabilistic atlases within FSLeyes. As these atlases do not include critical regions for numerical cognition (e.g., IPS), we used the Juelich Histological Atlas to label parietal regions. Only for data visualization, we extracted the beta values from the obtained clusters and then plotted against their respective covariate, including the corresponding correlation coefficient.

\section{Results}

\section{IPS Functional connectivity and mathematical ability}

\section{IPS functional connectivity is negatively associated with raw math ability in SPL}

Following Jolles, Ashkenazi, et al. (2016) correlational analyses, our first approach was to examine connectivity of the IPS as it relates raw math ability, measured by the WRAT computations scores as the covariate interest. At the stringent AFNI threshold, there were no regions showing a significant relationship between math ability and functional connectivity for 
any of the IPS seed ROIs. In contrast, at the less stringent extent threshold of $p<.01$, cluster size $=128$ voxels, (hereafter the Jolles (2016) threshold), we found that the functional connectivity of the left IPS showed a negative association with math ability. Specifically, the strength of the functional connectivity between the left IPS and the right SPL was negatively associated with the WRAT scores (Supplementary Figure 1 and Supplementary Table 1).

\section{Left IPS functional connectivity reveals a temporoccipital network negatively associated with math ability independent of IQ}

Given the strong correlation between math and IQ, we followed-up these analyses of the IPS functional connectivity by introducing the Math-IQ residuals as the covariate of interest. This analysis revealed that worse math performance was related to greater connectivity of the left IPS with two clusters (Figure 3A and Table 2): the larger with a peak in the right lateral occipital cortex extending to the fusiform gyrus and the hippocampus (Figure 3B, spatial extent 1463 voxels, $p<.05$ ) and the smaller with a peak in the left fusiform gyrus (Figure 3C, spatial extent 904 voxels, $p<.10$ ). Supplementary Figure 2 also shows the 11 clusters that showed functional connectivity with the left IPS at the less stringent Jolles (2016) threshold. At this threshold, there were 11 clusters that showed significant functional connectivity with IPS. Ten of them showed the same pattern of negative relations with math-IQ residuals (Supplementary Figure 2 and Supplementary Table 2).

As opposed to the functional connectivity of the left IPS, there were no significant clusters identified in the relationship between math ability and the functional connectivity of the right IPS, at the AFNI threshold. Only at the Jolles (2016) threshold did we observed functional connectivity of this region with five clusters (Supplementary Figure 3 and Supplementary Table

2). In all cases of cortical clusters, worse math performance was related to higher connectivity. 
Overall, these results indicate that stronger functional connectivity between the left IPS and bilateral occipital regions is found in adolescents who underachieve in math relative to their IQ scores and are consistent with other studies finding that IPS hyper-connectivity is associated with low math ability (Jolles, Ashkenazi, et al., 2016; Price et al., 2018)

Functional connectivity of hippocampus and math ability

No relations between raw math and hippocampal connectivity

We first examined the connectivity of the hippocampus related to raw math ability. At the AFNI threshold, there were no significant clusters for the relationship between math ability and the functional connectivity of the hippocampal seed ROIs.

Left hippocampus functional connectivity with frontal regions is negatively associated with math ability independent of IQ

The functional connectivity of the left hippocampus was also negatively associated with Math-IQ residuals. Specifically, two frontal clusters showed this pattern (Figure 4A and Table 3): the left pre-central gyrus (Figure 4B, spatial extent 909 voxels, $p<.10$ ) and another in the left insula, extending to the left IFG (Figure 4C, spatial extent 909 voxels, $p<.10$ ).

\section{Control Analyses}

Hippocampal functional connectivity is positively associated with individual differences in IQ

To assess the extent to which the negative associations between IPS and hippocampal functional connectivity were specific to math ability, we investigated whether the same pattern of results was observed with a measure of general cognitive ability, IQ. In contrast to the negative associations between individual differences in math ability and the functional connectivity of the IPS and hippocampus, individual differences in IQ showed a positive association with the functional connectivity, in this case of the left hippocampus (Figure 5A and Table 4). 
Specifically, adolescents with high IQ scores showed strong left hippocampal functional connectivity with one cluster with a peak in the IFG (Figure 5B, spatial extent 873 voxels, $p<$ .10). There was an overlap of 30 voxels in the left IFG between the hippocampal connectivity associated with IQ and the one associated with Math-IQ residuals. Critically, while the correlation of functional connectivity and IQ was positive, for the Math-IQ residuals the correlation was negative. Finally, there was no relation between IQ and right hippocampal connectivity, neither with the connectivity of the bilateral IPS.

\section{Math ability is only associated with functional connectivity of areas that are critical for math cognition}

To rule out the possibility that hyper-connectivity is a global feature of low math ability, we examined the association between the functional connectivity of a primary sensory region, Heschl's gyrus, and math ability. As expected, there were no significant clusters for the relationship between math achievement, neither when considering raw math scores nor Math-IQ residuals, and the functional connectivity of neither the right nor the left Heschl's gyrus. These control analyses support the hypothesis that hyper-connectivity of math cognition regions is a feature of low math ability in adolescence and provide evidence against a general pattern of hypo- or hyper-connectivity in low math ability.

\section{Discussion}

Recent research on the developmental changes in the task-based and resting-state functional connectivity related to math ability has started to point to a transition from negative to a positive relationship. That is, while in childhood, stronger resting-state functional connectivity of the IPS and other key brain regions for math cognition (e.g., angular gyrus, fusiform gyrus, hippocampus, and middle frontal gyrus) is associated with low math ability (Jolles, Supekar, et 
al., 2016; Price et al., 2018), in adulthood, stronger connectivity is related to better math ability (Koyama et al., 2017; Skagerlund et al., 2018). However, to date, no study has examined the resting-state functional connectivity related to math performance in adolescence. Using seedbased resting-state connectivity, in the current study, we demonstrated that IPS functional connectivity of 15- to 17-year-old adolescents was negatively related to math ability after controlling for IQ, resembling those patterns found in children. However, the specific regions connected with the IPS, a temporooccipital network, resembled those found in adult studies. Further, we demonstrated, for the first time, that contrary to studies of mathematical learning (Jolles, Ashkenazi, et al., 2016; Supekar et al., 2013), concurrent measures of math ability are negatively correlated with hippocampal functional connectivity. Importantly, brain areas that were functionally connected with the IPS and the hippocampus and were associated with math performance did not overlap with those that were associated with individual differences in general intelligence. Finally, our results also ruled-out hyper-connectivity as a global feature of low math performance, by demonstrating that connectivity of the Heschl's gyrus, a primary sensory region not involved to math cognition, was not related to math achievement. The current study fills a gap in our knowledge of the development of the neural correlates of math cognition by showing that adolescence is a transition stage in which patterns found in both childhood and adulthood are observed.

\section{IPS is hyper-connected in adolescents with low math ability}

Consistent with the negative association the IPS functional connectivity and math ability found in children (Jolles, Ashkenazi, et al., 2016; Price et al., 2018), in this study, adolescents with low math achievement relative to their IQ scores showed stronger IPS connectivity than their peers with high ability. However, during childhood, regions where hyper-connectivity with 
the IPS is associated with low math ability are the superior parietal lobule, the supramarginal gyrus, the superior temporal gyrus, and the anterior cingulate cortex (Jolles, Ashkenazi, et al., 2016). Here, instead, we found that low math ability was associated with the functional connectivity between the left IPS and a large right cluster, which extended from the lateral occipital cortex to the fusiform gyrus and the hippocampus, and a smaller left cluster, which included the left fusiform gyrus. Notably, at a lower threshold, we also detected hyperconnectivity of the IPS with supramarginal gyrus, anterior cingulate cortex, precentral gyrus, and planum polare, consistent with the results in childhood.

Developmental examinations of task activity and connectivity related to math ability converge on a shift in the recruitment of anterior regions to posterior brain areas (Houdé et al., 2010). For instance, when contrasting brain activity from children and adults in numerical tasks (Haist et al., 2015; Rivera et al., 2005) and recently inter-subject synchronization during a natural view task with math content (Kersey et al., 2019), studies report greater activity and synchronization in posterior regions (e.g., IPS and lateral occipital cortex) in adults than in children. Consistently, a longitudinal study found that task-based IPS functional connectivity shifts from frontal areas, like the dorsolateral and ventrolateral prefrontal cortex, to posterior regions, like the fusiform gyrus, in children from age 7 to 14 (Battista et al., 2018). Their working hypothesis was that the integration of the right fusiform gyrus, a brain region involved in visual number form processing, and the quantity processing system located in the parietal lobule, reflects the sophistication in mathematical problem-solving skills from early to late childhood (Battista et al., 2018). In the present study, while the direction of the effects was akin to that found in children, the specific regions were more consistent with studies of older children and adults. Thus, our results bring further support to this developmental hypothesis, by showing 
that in adolescents, regions that are functionally connected with the IPS (e.g., the fusiform gyrus and the lateral occipital cortex) and associated with differences in math performance resemble those found in studies with adults. Nevertheless, the negative direction of this association still resembles the one found in children.

Together, our results and previous research suggests that across both resting-state and task-based paradigms, a set of posterior regions (e.g., the IPS, the lateral occipital gyrus, and the fusiform gyrus) comprises the mature functional brain network associated with math performance, which can already be observed in adolescence.

\section{Hippocampal hyper-connectivity is related to concurrent low math ability in adolescence}

Although traditional brain-based models of numerical cognition do not consider the contributions of the hippocampus (e.g., Dehaene et al., 2003), more recent theorizing has started to recognize its role from a task perspective (Kucian, 2016; Menon, 2016). With regards to resting state, hippocampal connectivity has not been investigated in connection with individual differences in math skills, instead the focus has been limited to examining its predictive power to learning outcomes and changes related to learning. For instance, Supekar et al. (2013) found that greater gains in performance after an intensive arithmetic intervention is related to greater hippocampal resting-state connectivity with frontal regions, including dorsolateral and ventrolateral prefrontal cortex and supplementary motor area, and middle temporal gyrus. Similarly, children who display greater positive changes in their efficiency to solve arithmetic problems, after the same intervention, strengthened their IPS connectivity with the bilateral hippocampus (Jolles, Supekar, et al., 2016). These results suggest that as a consequence of efficient math learning ability, stronger hippocampal resting-state connectivity may be associated with better contemporaneous math skills. Here, we tested this hypothesis by examining for the 
first time the relationship between hippocampal functional connectivity and concurrent math skills. At odds with this hypothesis, our results indicate that stronger hippocampal connectivity with two clusters (the pre-central gyrus and the insula) was associated with worse, not better, math ability.

How can we reconcile the current finding of stronger connectivity associated with lower math skills with previous studies showing the opposite pattern for math learning, that is stronger connectivity associate with greater learning? One possible explanation for the divergence in the directions of these two relationships is that the hippocampal networks involved during math learning might be different from those associated with individual differences in math achievement. However, the regions that we report in this study (the precentral gyrus and the insula) are consistent with previous hippocampal task-based and resting-state connectivity research. For instance, greater right hippocampal connectivity with the supplementary motor area, during rest, predicts math learning outcomes (Supekar et al., 2013). In task-based studies, activity of the precentral gyrus is related to finger-count strategies (Kaufmann et al., 2011). There is a need for further evidence about the role the precentral gyrus in math cognition during adolescence, included the possibility that very low achieving students may still be using fingercounting.

Greater task-based connectivity between the right hippocampus and anterior insula is thought to play a key role in the transition from counting-based procedures to fact-retrieval strategies (Cho et al., 2012). Similarly, insular intrinsic connectivity with premotor cortex is related to children's subtraction skills, while insular connectivity with supramarginal gyrus and frontal regions was related to multiplication skills (Chang et al., 2018). Overall, although our findings are at odds with the reported direction of these past studies, the brain regions we found 
are consistent with those proposed as part of the hippocampal-frontal declarative memory system, which is comprised by the anterior insula and the ventral and dorsolateral prefrontal cortex and contributes to the maturation of math retrieval skills (Menon, 2016).

A second possibility is a developmental shift in the direction of the hippocampal functional connectivity. Contrary to the observed patterns in the IPS functional connectivity, which is consistently negative, the relationship between hippocampal resting-state connectivity and math ability might shift from being positive during childhood (Jolles, Supekar, et al., 2016; Supekar et al., 2013) to negative in later stages of development. This interpretation finds support in the decrease of hippocampal activity later in development (Qin et al., 2014; Rivera et al., 2005). For instance, left hippocampal activity while performing an arithmetic task is negatively correlated with age (Rivera et al., 2005), and children that had recently acquired retrieval strategies showed hippocampal activity while solving addition problems but adolescents and adults do not (Qin et al., 2014). Together, these results suggest that the contributions of the hippocampus might be circumscribed to contexts of math learning, particularly during childhood; thus, lower hippocampal connectivity during adolescence might be a sign of hippocampal networks achieving the adult pattern, while strong connectivity might indicate that they are still under development.

Finally, a third non-mutually exclusive explanation is the different math measures employ across studies. Studies that have reported a positive correlation between hippocampal resting-state connectivity and math learning have only used non-standardized measures of a single math ability (e.g., improvement in addition problems). In contrast, studies that have reported negative correlations of task-based and resting-state functional connectivity of other brain regions, like the IPS, have used standardized measures of math ability (Jolles, Ashkenazi, 
et al., 2016; Michels et al., 2018). Standardized timed math measures (e.g., math fluency) and untimed progressive measures typically parallel arithmetic skills in early elementary school. Only in later grades to measures diverge, with untimed measures incorporating more complex mathematical domains (e.g., rational numbers, algebra, geometry). Thus, there is a call for future studies to investigate whether different measures of math ability show differential patterns of functional connectivity across development.

\section{Different connectivity patterns and networks are associated with IQ than those associated} with math ability

Research in mathematical disability (MD) has strived to disentangle the effects of IQ from those of the learning disability per se (Dennis et al., 2009) given the well-established positive relationship between individual differences in intelligence and math outcomes. In fact, a recent meta-analysis reported a reliable association between fluid intelligence and mathematical ability $(r=.41)$ (Peng et al., 2019). To address this issue in resting-state research, authors either match participants with MD and typically developing children by IQ scores (Jolles, Ashkenazi, et al., 2016) or used IQ as a covariate (Price et al., 2018). Consistent with these prior results, we found that after controlling for IQ, we observed that IPS and hippocampal functional connectivity was negatively associated with math performance, although the literature finds positive results as well (Skagerlund et al., 2018). In contrast to this variability, the direction of the relationship of individual differences in intelligence and intrinsic functional connectivity is consistently reported as positive (Hearne et al., 2016). Brain networks related to intelligence are also comprised of regions of the frontoparietal and default mode networks (Dubois et al., 2018), not unlike those that have been related to math ability. 
As our most robust results were found when regressing out IQ, an outstanding question arose whether the brain networks negatively associated with math ability discrepancy, were simply the networks positively related to IQ. To rule out this possibility, we examined the functional connectivity of IQ on its own. Consistent with prior research, we found that functional connectivity correlated positively with general intelligence. In particular, the left hippocampal functional connectivity with a cluster in the inferior frontal gyrus was positively associated with IQ scores. This cluster extended to the middle frontal gyrus, which comprises the frontoparietal network. In contrast, only a small portion of this cluster overlapped with clusters associated with math ability. Specifically, this overlap comprised 30 voxels of the inferior frontal gyrus and was between the left hippocampal connectivity related to IQ scores and that of math ability. Furthermore, when we examined raw math ability, the only cluster found for the association between IPS functional connectivity was negative, suggesting that the negative association between functional connectivity and math-IQ residuals is not an artifact of the regressing out IQ. Together, these results converge on the contention that brain networks dedicated to math ability and general intelligence are not only are anatomically distinct but also differ in their directionality.

Why is stronger connectivity associated with both high intelligence and low math ability? Aside from anatomical differences comprising the math and intelligence networks, it has been suggested that hyper-connectivity associated with intelligence may allow maintaining a large repertoire of neural states at rest, which in turn will facilitate flexibly adjusting to task demands (Hearne et al., 2016). In contrast, hyper-connectivity during rest may hinder the ability to engage and modulate brain circuits that are relevant to solve mathematical problems (Jolles, Ashkenazi, et al., 2016; Rosenberg-Lee et al., 2015). 


\section{Considerations and future directions}

As the field of developmental cognitive neuroscience moves from lenient parametric cluster-based thresholding methods to highly stringent non-parametric approaches (Flournoy et al., 2020), a challenge is introduced in comparing newer studies to older work. Here, to balance the concerns about type I and type II errors and to facilitate comparison of our results with those presented in Jolles et al. (2016), we employed a more lenient than recommended alpha-value for our cluster-extent threshold $(p<.10)$, recommended (Eklund et al., 2016). The reported clusters were relatively large (greater than $9002 \mathrm{~mm}^{3}$ voxels), indeed, larger than those previously reported (Jolles, Ashkenazi, et al., 2016). Crucially, essentially all the clusters (and those at the very lenient Jolles (2016)), all showed the same direction of effect, namely hyper-connectivity was associated with poor math achievement.

Given the relatively small sample size, our study did not have sufficient power to detect effects with small to medium effect sizes. Therefore, other brain areas may have smaller contributions to math achievement during adolescence but require larger samples to be detected. Furthermore, as prevalence for math disability and talent correspond to less than $10 \%$ for each condition (Reigosa-Crespo et al., 2012; Warrier \& Baron-Cohen, 2016), research with these types of samples is often underpowered. Yet, more reliable conclusions could be reached based on the cumulative output of multiple studies with small sample sizes (Klapwijk et al., 2021). These concerns highlight the importance of replication studies and point to the potential of reanalyses with aggregated data from multiple studies. Indeed, this aggregation approach has proved fruitful in resting-state fMRI research examining individuals with autism (Di Martino et al., 2014) and attention deficit hyperactivity disorder (Bellec et al., 2017), providing a promising 
template for efforts to understand variation in math achievement across the ability spectrum (current data available upon request).

Finally, one of the main analytical challenges of resting-state analyses is choosing and defining ROI seeds. Here, we chose two regions (IPS and hippocampus) that child research has pointed out as pivotal for math achievement and learning. However, we omitted other regions, most notably the dorsolateral prefrontal cortex, that has also been highlighted by network models of math cognition (Kucian, 2016; Menon, 2010) and meta-analyses (Arsalidou \& Taylor, 2011; Sokolowski et al., 2017). Given the scarcity of research into the intrinsic connectivity of this area (only Koyama et al., (2017) has considered it in adults), tracking its developmental trajectory remains an important open question. Beyond seed-based analyses, future work employing multivariate and graph-theory analyses will afford more data-driven examinations of connectivity between and across networks (e.g., H. Zhang et al., 2019).

\section{Conclusions}

Studies looking at developmental changes in the task-based and resting-state functional connectivity related to math ability have started to point to a transition from a negative to a positive relationship. However, as research on math cognition has mostly overlooked adolescence, there was a gap in our knowledge on how the neural correlates of math achievement change during the transition from childhood to adulthood. Here, we found that in adolescents, the direction for the relationship between the IPS connectivity and math performance was similar to that found in children, but the specific regions were consistent with those reported in adults. Further, math achievement has a negative relationship with hippocampal functional connectivity. Together, our study suggests that hyper-connectivity during adolescence still is a hallmark of low 
math ability, which is not exclusively to the IPS, but also present in other areas critical for math cognition. 


\section{References}

Abreu-Mendoza, R. A., Chamorro, Y., \& Matute, E. (2019). Psychometric Properties of the WRAT Math Computation Subtest in Mexican Adolescents. Journal of Psychoeducational Assessment, 37(8), 957-972. https://doi.org/10.1177/0734282918809793

Abreu-Mendoza, R. A., Zarabozo-Hurtado, D., Chamorro, Y., Vazquez, P., Matute, E., \& Fandakova, Y. (2020). The Neural Correlates of the Core Number Systems Contribute to Mathematical Ability in Adolescence. PsyArXiv. https://doi.org/10.31234/osf.io/96tuy American Psychiatric Association. (2013). Diagnostic and statistical manual of mental disorders (DSM-5®). American Psychiatric Pub.

Arsalidou, M., \& Taylor, M. J. (2011). Is $2+2=4$ ? Meta-analyses of brain areas needed for numbers and calculations. NeuroImage, 54, 2382-2393.

https://doi.org/10.1016/j.neuroimage.2010.10.009

Battista, C., Evans, T. M., Ngoon, T. J., Chen, T., Chen, L., Kochalka, J., \& Menon, V. (2018). Mechanisms of interactive specialization and emergence of functional brain circuits supporting cognitive development in children. Npj Science of Learning, 3(1). https://doi.org/10.1038/s41539-017-0017-2

Bellec, P., Chu, C., Chouinard-Decorte, F., Benhajali, Y., Margulies, D., \& Craddock, R. C. (2017). The Neuro Bureau ADHD-200 Preprocessed Repository. NeuroImage, 144, 037044. https://doi.org/10.1101/037044

Chang, T. T., Lee, P. H., \& Metcalfe, A. W. S. (2018). Intrinsic insula network engagement underlying children's reading and arithmetic skills. NeuroImage, 167(64), 162-177. https://doi.org/10.1016/j.neuroimage.2017.11.027

Cho, S., Metcalfe, A. W. S., Young, C. B., Ryali, S., Geary, D. C., \& Menon, V. (2012). 
Hippocampal-prefrontal engagement and dynamic causal interactions in the maturation of children's fact retrieval. Journal of Cognitive Neuroscience, 24(9), 1849-1866. https://doi.org/10.1162/jocn_a_00246

Cho, S., Ryali, S., Geary, D. C., \& Menon, V. (2011). How does a child solve 7+8? Decoding brain activity patterns associated with counting and retrieval strategies. Developmental Science, 14(5), 989-1001. https://doi.org/10.1111/j.1467-7687.2011.01055.x

Choi, H. J., Zilles, K., Mohlberg, H., Schleicher, A., Fink, G. R., Armstrong, E., \& Amunts, K. (2006). Cytoarchitectonic identification and probabilistic mapping of two distinct areas within the anterior ventral bank of the human intraparietal sulcus. Journal of Comparative Neurology, 495(1), 53-69. https://doi.org/10.1002/cne.20849

Cole, M. W., Bassett, D. S., Power, J. D., Braver, T. S., \& Petersen, S. E. (2014). Intrinsic and task-evoked network architectures of the human brain. Neuron, 83(1), 238-251. https://doi.org/10.1016/j.neuron.2014.05.014

Cox, R. W. (1996). AFNI: Software for analysis and visualization of functional magnetic resonance neuroimages. Computers and Biomedical Research, 29(3), 162-173. https://doi.org/10.1006/cbmr.1996.0014

Cox, R. W., Chen, G., Glen, D. R., Reynolds, R. C., \& Taylor, P. A. (2017). FMRI Clustering in AFNI: False-Positive Rates Redux. Brain Connectivity, 7(3), 152-171. https://doi.org/10.1089/brain.2016.0475

Dehaene, S., \& Cohen, L. (2007). Cultural recycling of cortical maps. Neuron, 56, 384. papers3://publication/uuid/F4A19C17-EE1D-4941-8869-9D73D052DA1B

Dehaene, S., Piazza, M., Pinel, P., \& Cohen, L. (2003). Three parietal circuits for number processing. Conitive Neuropsychology, 20, 487-506. 
Dennis, M., Francis, D. J., Cirino, P., Schachar, R., Barnes, M. A., \& Fletcher, J. (2009). Why IQ is not covariate in cognitive studies of neurodevelopmental disorers. Journal of the International Neuropsychological Society, 15, 331-343.

Di Martino, A., Yan, C. G., Li, Q., Denio, E., Castellanos, F. X., Alaerts, K., Anderson, J. S., Assaf, M., Bookheimer, S. Y., Dapretto, M., Deen, B., Delmonte, S., Dinstein, I., ErtlWagner, B., Fair, D. A., Gallagher, L., Kennedy, D. P., Keown, C. L., Keysers, C., ... Milham, M. P. (2014). The autism brain imaging data exchange: Towards a large-scale evaluation of the intrinsic brain architecture in autism. Molecular Psychiatry, 19(6), 659667. https://doi.org/10.1038/mp.2013.78

Dubois, J., Galdi, P., Paul, L. K., \& Adolphs, R. (2018). A distributed brain network predicts general intelligence from resting-state human neuroimaging data. Philosophical Transactions of the Royal Society B: Biological Sciences, 373(1756). https://doi.org/10.1098/rstb.2017.0284

Eklund, A., Nichols, T. E., \& Knutsson, H. (2016). Cluster failure: Why fMRI inferences for spatial extent have inflated false-positive rates. Proceedings of the National Academy of Sciences of the United States of America, 113(28), 7900-7905.

https://doi.org/10.1073/pnas.1602413113

Fias, W., Menon, V., \& Szucs, D. (2013). Multiple components of developmental dyscalculia. Trends in Neuroscience and Education, 2(2), 43-47. https://doi.org/10.1016/j.tine.2013.06.006

Flournoy, J. C., Vijayakumar, N., Cheng, T. W., Cosme, D., Flannery, J. E., \& Pfeifer, J. H. (2020). Improving practices and inferences in developmental cognitive neuroscience. Developmental Cognitive Neuroscience, 45. https://doi.org/10.1016/j.den.2020.100807 
Forman, S. D., Cohen, J. D., Fitzgerald, M., Eddy, W. F., Mintun, M. A., \& Noll, D. C. (1995). Improved Assessment of Significant Activation in Functional Magnetic Resonance Imaging (fMRI): Use of a Cluster-Size Threshold. Magnetic Resonance in Medicine, 33(5), 636647. https://doi.org/10.1002/mrm.1910330508

Fox, M. D., \& Raichle, M. E. (2007). Spontaneous fluctuations in brain activity observed with functional magnetic resonance imaging. Nature Reviews Neuroscience, 8(9), 700-711. https://doi.org/10.1038/nrn2201

Gerardi, K., Goette, L., \& Meier, S. (2013). Numerical ability predicts mortgage default. Proceedings of the National Academy of Sciences, 110(28), 11267-11271. https://doi.org/10.1073/pnas.1220568110

Haist, F., Wazny, J. H., Toomarian, E., \& Adamo, M. (2015). Development of brain systems for nonsymbolic numerosity and the relationship to formal math academic achievement. Human Brain Mapping, 36(2), 804-826.

Handel, M. J. (2016). What do people do at work? Journal for Labour Market Research, 49(2), 177-197. https://doi.org/10.1007/s12651-016-0213-1

Hearne, L. J., Mattingley, J. B., \& Cocchi, L. (2016). Functional brain networks related to individual differences in human intelligence at rest. Scientific Reports, 6(August), 1-8. https://doi.org/10.1038/srep32328

Houdé, O., Rossi, S., Lubin, A., \& Joliot, M. (2010). Mapping numerical processing, reading, and executive functions in the developing brain: An fMRI meta-analysis of 52 studies including 842 children. Developmental Science, 13(6), 876-885. https://doi.org/10.1111/j.1467-7687.2009.00938.x

Iuculano, T., Rosenberg-Lee, M., Richardson, J., Tenison, C., Fuchs, L., Supekar, K., \& Menon, 
V. (2015). Cognitive tutoring induces widespread neuroplasticity and remidates brain function in children with mathematical learning disabilities. Nature Communications, 6(8453). https://doi.org/10.1038/ncomms9453

Jolles, D., Ashkenazi, S., Kochalka, J., Evans, T., Richardson, J., Rosenberg-Lee, M., Chen, T., \& Menon, V. (2016). Parietal hyper-connectivity, aberrant brain organization, and circuitbased biomarkers in children with mathematical disabilities. Developmental Science. https://doi.org/10.1111/desc.12399

Jolles, D., Supekar, K., Richardson, J., Tenison, C., Ashkenazi, S., Rosenberg-Lee, M., Fuchs, L., \& Menon, V. (2016). Reconfiguration of parietal circuits with cognitive tutoring in elementary school children. Cortex, 83, 231-245.

https://doi.org/10.1016/j.cortex.2016.08.004

Jung, R. E., \& Haier, R. J. (2007). The Parieto-Frontal Integration Theory (P-FIT) of intelligence: Converging neuroimaging evidence. Behavioral and Brain Sciences, 30(2), 135-154. https://doi.org/10.1017/S0140525X07001185

Kaufman, L., Wood, G., Rubistein, O., \& Henik, A. (2011). Meta-Analyses of Developmental fMRI Studies Investigating Typical and Atypical Trajectories of Number Processing and Calculation. Developmental Neuropsychology, 36(6), 763-787.

Kaufmann, L., Wood, G., Rubinsten, O., \& Henik, A. (2011). Meta-analyses of developmental fMRI studies investigating typical and atypical trajectories of number processing and calculation. Developmental Neuropsychology, 36(6), 763-787. https://doi.org/10.1080/87565641.2010.549884

Kersey, A. J., Wakim, K. M., Li, R., \& Cantlon, J. F. (2019). Developing, mature, and unique functions of the child's brain in reading and mathematics. Developmental Cognitive 
Neuroscience, 39(July), 100684. https://doi.org/10.1016/j.den.2019.100684

Klapwijk, E. T., van den Bos, W., Tamnes, C. K., Raschle, N. M., \& Mills, K. L. (2021).

Opportunities for increased reproducibility and replicability of developmental neuroimaging. Developmental Cognitive Neuroscience, 47.

https://doi.org/10.1016/j.den.2020.100902

Klein, E., Moeller, K., Glauche, V., Weiller, C., \& Willmes, K. (2013). Processing Pathways in Mental Arithmetic-Evidence from Probabilistic Fiber Tracking. PLoS ONE, 8(1). https://doi.org/10.1371/journal.pone.0055455

Kovas, Y., Giampietro, V., Viding, E., Ng, V., Brammer, M., Barker, G., Happé, F. G. E., \& Plomin, R. (2009). Brain Correlates of Non-Symbolic Numerosity Estimation in Low and High Mathematical Ability Children. PloS ONE, 4(2), e4587.

Koyama, M. S., Molfese, P. J., Milham, M. P., Mencl, W. E., \& Pugh, K. R. (2020). Thalamus is a common locus of reading, arithmetic, and IQ: Analysis of local intrinsic functional properties. Brain and Langauge, 209, 1-14.

Koyama, M. S., O’Connor, D., Shehzad, Z., \& Milham, M. P. (2017). Differential contributions of the middle frontal gyrus functional connectivity to literacy and numeracy. Scientific Reports, 7(1), 1-13. https://doi.org/10.1038/s41598-017-17702-6

Kucian, K. (2016). Developmental dyscalculia and the brain. In Development of Mathematical Cognition (pp. 165-193). Elsevier. https://doi.org/10.1016/b978-0-12-801871-2.00007-1

Matute, E., Rosselli, M., Ardila, A., \& Ostrosky, F. (2007). Evaluación Neuropsicológica Infantil. Manual Moderno.

McCaskey, U., von Aster, M., O’Gorman Tuura, R., \& Kucian, K. (2017). Adolescents with developmental dyscalculia do not have a generalized magnitude deficit - Processing of 
discrete and continuous magnitudes. Frontiers in Human Neuroscience, 11(March), 1-19. https://doi.org/10.3389/fnhum.2017.00102

Menon, V. (2010). Developmental cognitive neuroscience of arithmetic: Implications for learning and education. ZDM - International Journal on Mathematics Education, 42(6), 515-525. https://doi.org/10.1007/s11858-010-0242-0

Menon, V. (2016). Memory and cognitive control circuits in mathematical cognition and learning. Progress in Brain Research, 227, 159-186.

https://doi.org/10.1016/bs.pbr.2016.04.026

Michels, L., O'Gorman, R., \& Kucian, K. (2018). Functional hyperconnectivity vanishes in children with developmental dyscalculia after numerical intervention. Developmental Cognitive Neuroscience, 30(March 2017), 291-303.

https://doi.org/10.1016/j.den.2017.03.005

Myers, T., Carey, E., \& Szücs, D. (2017). Cognitive and Neural Correlates of Mathematical Giftedness in Adults and Children: A Review. Frontiers in Psychology, 8(1646). https://doi.org/10.3389/fpsyg.2017.01646

Peng, P., Wang, T., Wang, C. C., \& Lin, X. (2019). A meta-analysis on the relation between fluid intelligence and reading/ mathematics: Effects of tasks, age, and social economics status. Psychological Bulletin, 145(2), 189-236. https://doi.org/10.1037/bul0000182

Power, J. D., Barnes, K. A., Snyder, A. Z., Schlaggar, B. L., \& Petersen, S. E. (2012). Spurious but systematic correlations in functional connectivity MRI networks arise from subject motion. NeuroImage, 59(3), 2142-2154. https://doi.org/10.1016/j.neuroimage.2011.10.018

Price, G. R., Holloway, I., Räsänen, P., Vesterinen, M., \& Ansari, D. (2007). Impaired parietal magnitude processing in developmental dyscalculia. Current Biology, 17(24), R1042- 
R1043.

Price, G. R., Yeo, D. J., Wilkey, E. D., \& Cutting, L. E. (2018). Prospective relations between resting-state connectivity of parietal subdivisions and arithmetic competence.

Developmental Cognitive Neuroscience, 30, 280-290.

https://doi.org/10.1016/j.den.2017.02.006

Qin, S., Cho, S., Chen, T., Rosenberg-lee, M., Geary, D. C., \& Menon, V. (2014). Hippocampalneocortical functional reorganization underlies children's cognitive development. Nature Neuroscience. https://doi.org/10.1038/nn.3788

Reigosa-Crespo, V., Valdés-Sosa, M., Butterworth, B., Estévez, N., Rodríguez, M., Santos, E., Torres, P., Suárez, R., \& Lage, A. (2012). Basic numerical capacities and prevalence of developmental dyscalculia: The Havana survey. Developmental Psychology, 48(1), 123135. https://doi.org/10.1037/a0025356

Reyna, V. F., Nelson, W. L., Han, P. K., \& Dieckmann, N. F. (2009). How Numeracy Influences Risk Comprehension and Medical Decision Making. Psychological Bulletin, 135(6), 943973.

Ritchie, S. J., \& Bates, T. C. (2013). Enduring Links From Childhood Mathematics and Reading Achievement to Adult Socioeconomic Status. Psychological Science, 24(7), 1301-1308.

Rivera, S. M., Reiss, A. L., Eckert, M. A., \& Menon, V. (2005). Developmental changes in mental arithmetic: Evidence for increased functional specialization in the left inferior parietal cortex. Cerebral Cortex, 15(11), 1779-1790. https://doi.org/10.1093/cercor/bhi055

Rolls, E. T., Huang, C. C., Lin, C. P., Feng, J., \& Joliot, M. (2020). Automated anatomical labelling atlas 3. NeuroImage, 206(August 2019), 116189. https://doi.org/10.1016/j.neuroimage.2019.116189 
Rosenberg-Lee, M., Ashkenazi, S., Chen, T., Young, C. B., Geary, D. C., \& Menon, V. (2015). Brain hyper-connectivity and operation-specific deficits during arithmetic problem solving in children with developmental dyscalulia. Developmental Science, 18(3), 351-372.

Rosenberg-Lee, M., Barth, M., \& Menon, V. (2011). What difference does a year of schooling make?. Maturation of brain response and connectivity between 2nd and 3rd grades during arithmetic problem solving. Neurolmage, 57(3), 796-808. https://doi.org/10.1016/j.neuroimage.2011.05.013

Rosenberg-Lee, M., Iuculano, T., Bae, S. R., Richardson, J., Qin, S., Jolles, D., \& Menon, V. (2018). Short-term cognitive training recapitulates hippocampal functional changes associated with one year of longitudinal skill development. Trends in Neuroscience and Education, 10(December 2017), 19-29. https://doi.org/10.1016/j.tine.2017.12.001

Sattler, J. (2010). Evaluación infantil: Fundamentos cognitivos: Vol. I (5ta ed.). Manual Moderno.

Scheperjans, F., Hermann, K., Eickhoff, S. B., Amunts, K., Schleicher, A., \& Zilles, K. (2008). Observer-independent cytoarchitectonic mapping of the human superior parietal cortex. Cerebral Cortex, 18(4), 846-867. https://doi.org/10.1093/cercor/bhm116

Schneider, M., Beeres, K., Coban, L., Merz, S., Schmidt, S., Stricker, J., \& De Smedt, B. (2017). Associations of non-symbolic and symbolic numerical magnitude processing with mathematical competence: A meta-analysis. Developmental Science, 20, e12372.

Skagerlund, K., Bolt, T., Nomi, J. S., Skagenholt, M., Västfjäll, D., Träff, U., \& Uddin, L. Q. (2018). Disentangling mathematics from executive functions by investigating unique functional connectivity patterns predictive of mathematics ability. Journal of Cognitive Neuroscience, 31(4), 560-573. https://doi.org/10.1162/jocn_a_01367 
Sokolowski, H. M., Fias, W., Mousa, A., \& Ansari, D. (2017). Common and distinct brain regions in both parietal and frontal cortex support symbolic and nonsymbolic number processing in humans: A functional neuroimaging meta-analysis. NeuroImage, 146, 376394.

Supekar, K., Swigart, A. G., Tenison, C., Jolles, D. D., Rosenberg-Lee, M., Fuchs, L., \& Menon, V. (2013). Neural predictors of individual differences in response to math tutoring in primary-grade school children. Proceedings of the National Academy of Sciences of the United States of America, 110(20), 8230-8235. https://doi.org/10.1073/pnas.1222154110

Van Den Heuvel, M. P., \& Pol, H. E. H. (2010). Exploring the brain network: A review on resting-state fMRI functional connectivity. European Neuropsychopharmacology, 20(8), 519-534. https://doi.org/https://doi.org/10.1016/j.euroneuro.2010.03.008

Van Dijk, K. R. A., Hedden, T., Venkataraman, A., Evans, K. C., Lazar, S. W., \& Buckner, R. L. (2010). Intrinsic functional connectivity as a tool for human connectomics: Theory, properties, and optimization. Journal of Neurophysiology, 103(1), 297-321. https://doi.org/10.1152/jn.00783.2009

Ward, D. B. (2000). Simultaneous Inference for fMRI Data.

Warrier, V., \& Baron-Cohen, S. (2016). Genetics of Mathematical Aptitude. ELS, 1-6. https://doi.org/10.1002/9780470015902.a0026848

Wechsler, D. (2007). Escala Wechsler de Inteligencia para Niños-IV. Manual Moderno.

Wilkinson, G. S., \& Robertson, G. J. (2006). WRAT 4 Wide Range Achievement Test. Psychological Assessment Resources.

Zhang, H., Wee, C. Y., Poh, J. S., Wang, Q., Shek, L. P., Chong, Y. S., Fortier, M. V., Meaney, M. J., Broekman, B. F. P., \& Qiu, A. (2019). Fronto-parietal numerical networks in relation 
with early numeracy in young children. Brain Structure and Function, 224(1), 263-275. https://doi.org/10.1007/s00429-018-1774-2

Zhang, Y., Brady, M., \& Smith, S. (2001). Segmentation of brain MR images through a hidden Markov random field model and the expectation-maximization algorithm. IEEE

Transactions on Medical Imaging, 20(1), 45-57. https://doi.org/10.1109/42.906424 
A)
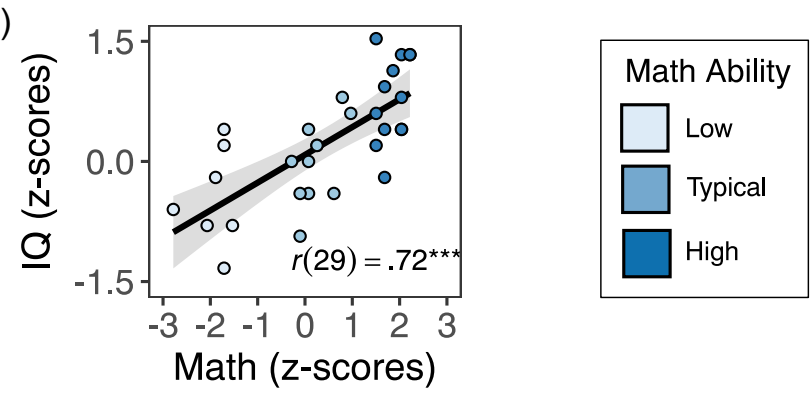

B)
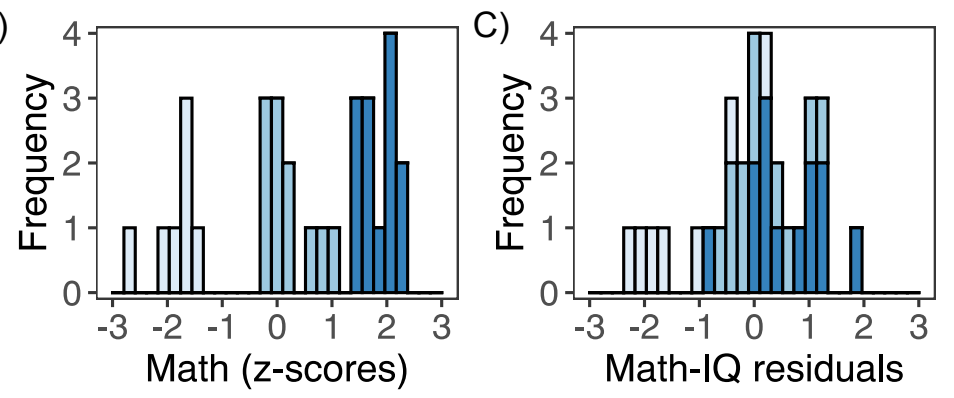

Figure 1. Performance on IQ and math achievement measures. A) IQ was strongly correlated with correct responses in the WRAT Math Computations subtest $(r(29)=.72, p<$ .001 . B) As participants were originally recruited to form part of one of three groups with nonoverlapping mathematical achievement levels, WRAT Math Computation z-scores were not normally distributed ( $\mathrm{W}=.90, p=.006)$. C) To control for the effect of IQ, we obtained the residuals of the linear regression when using IQ as the predictor and WRAT Math Computation scores as the dependent variable. Math-IQ Residuals were normally distributed $(\mathrm{W}=.95, p=$ $.210)$. 


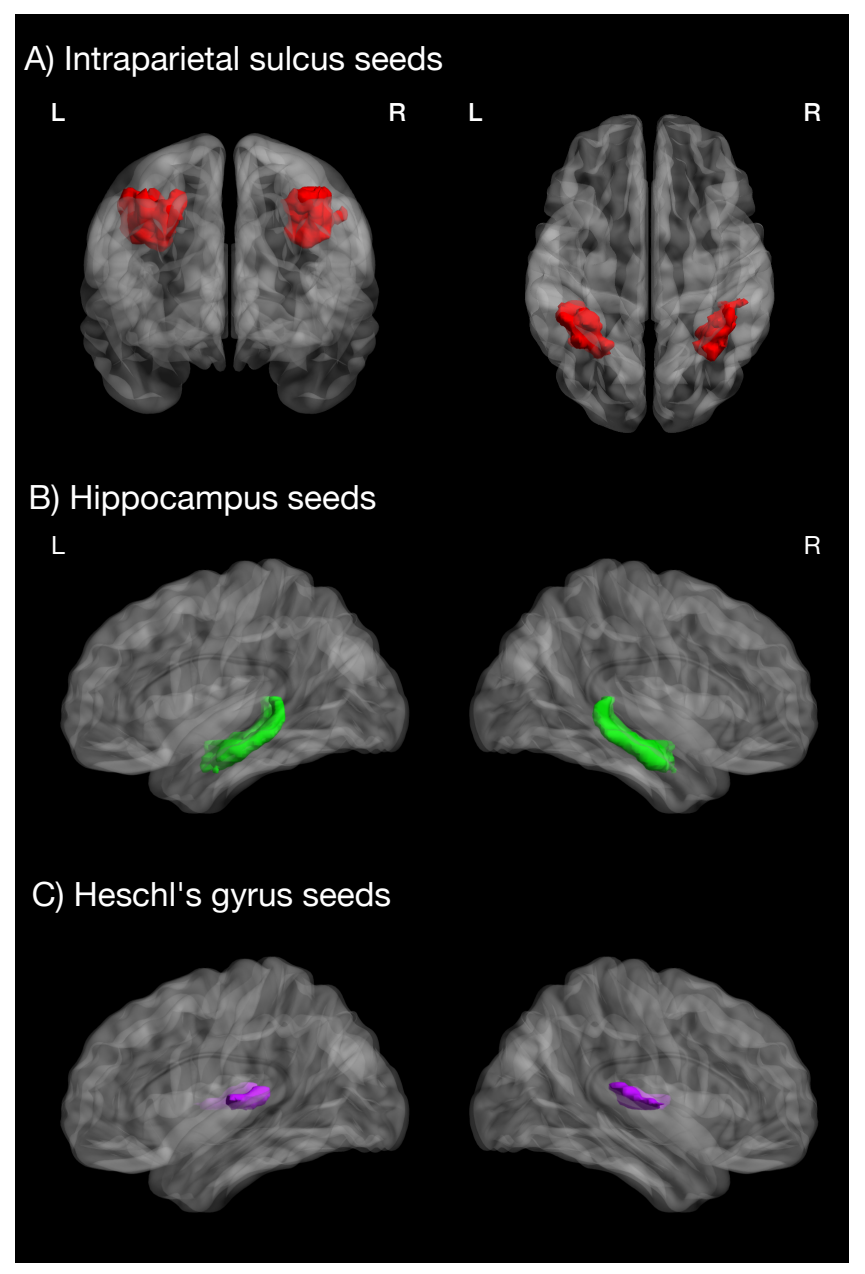

Figure 2. Anatomical seed regions of interest (ROI) and control seed region. A)

Intraparietal sulcus (IPS) ROIs were defined by combining the three IPS subdivision from the Juelich histological atlas (Choi et al., 2006; Scheperjans et al., 2008). B) Hippocampus ROIs were defined using the Anatomical Automatic Labeling (AAL) atlas (Rolls et al., 2020). C) Heschl's gyrus was used as the control seed region, similar to Jolles, Supekar et al. (2016), as was also defined using the AAL atlas. 


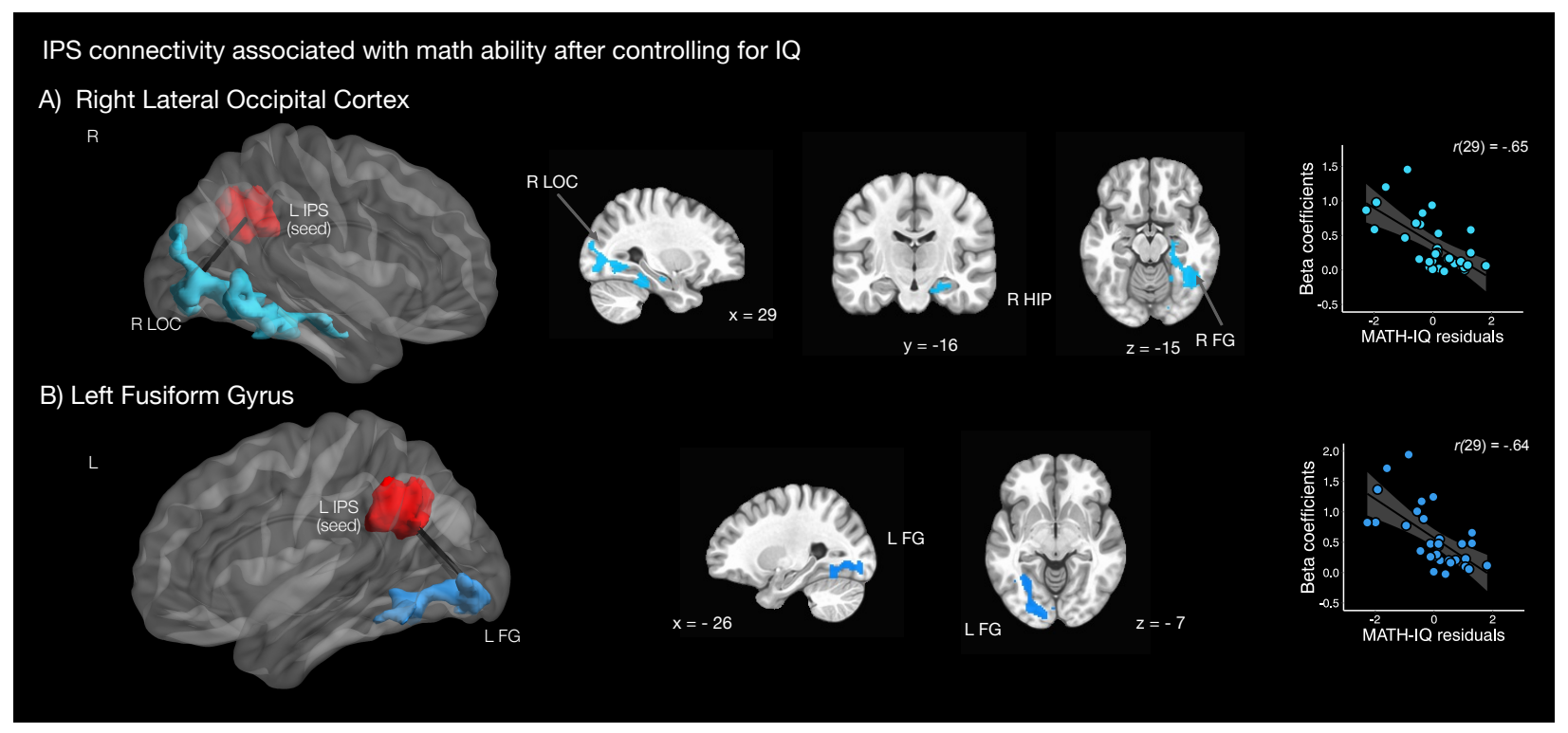

Figure 3. Left IPS functional connectivity associated with mathematical performance controlling for IQ. Low Math-IQ residuals were characterized by a strong IPS functional connectivity with A) a cluster in the right LOC (height threshold $p<.01$, extent threshold $p<$ .05 ), which extended to the HIP, and FG, and B) a cluster in the left FG (height threshold $p<$ .01 , extent threshold $p<.10$ ). Note. $\mathrm{L}=$ left, $\mathrm{FG}=$ fusiform gyrus, $\mathrm{HIP}=$ hippocampus, IPS = intraparietal sulcus, $\mathrm{LOC}=$ lateral occipital cortex. Scatterplots are presented for illustrative purposes. 


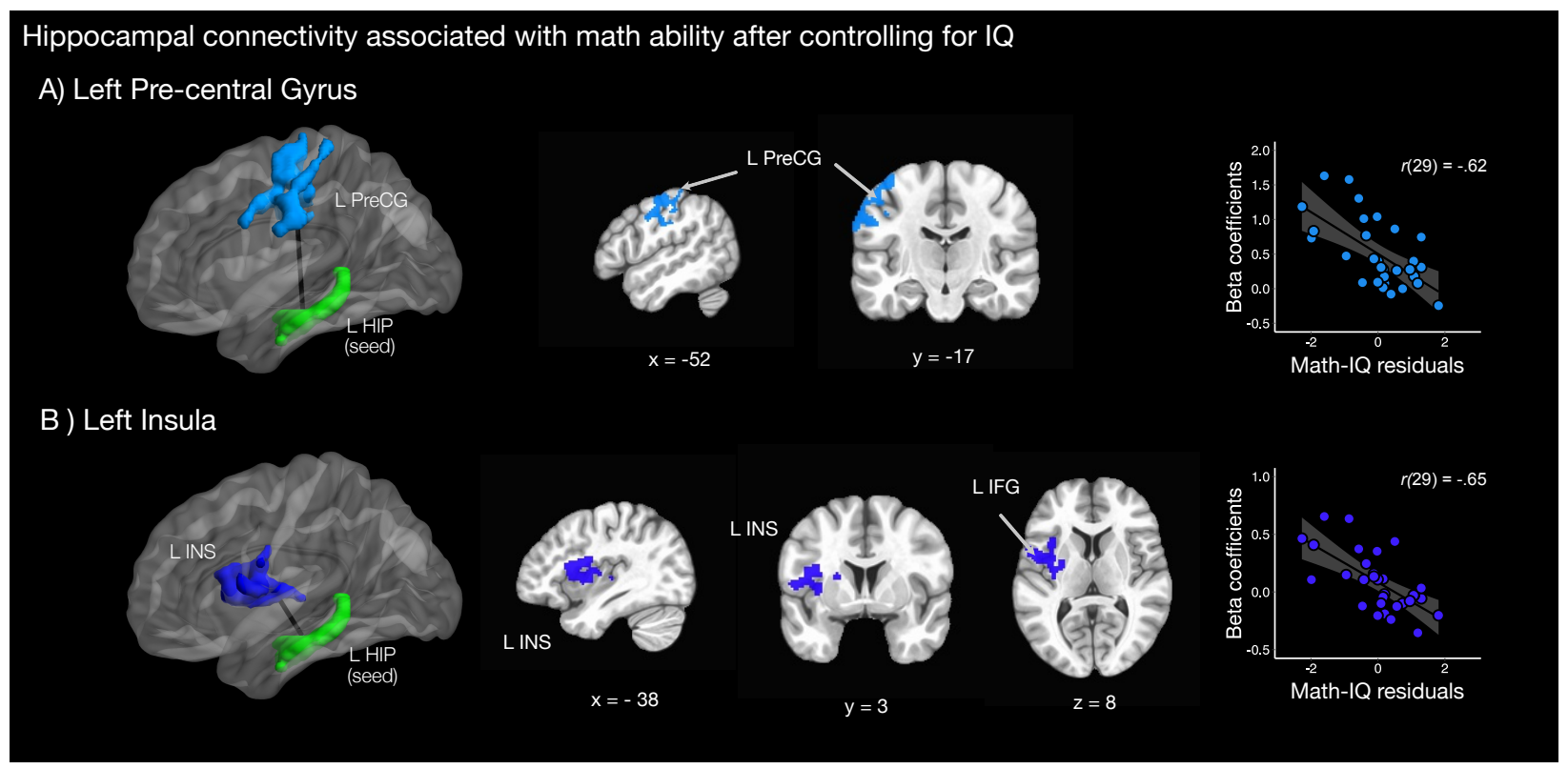

Figure 4. Left hippocampal functional connectivity associated with mathematical performance after controlling for IQ. Low Math-IQ residuals were characterized by a strong hippocampal functional connectivity with A) a cluster in the left preCG (height threshold $p<.01$, extent threshold $p<.10$ ), and $\mathrm{B}$ ) a cluster in the left insula (height threshold $p<.01$, extent threshold $p<.10$ ), which extended to the IFG. Note. $\mathrm{L}=$ left, HIP = hippocampus, IFG = inferior frontal gyrus, INS = insula, preCG = precentral gyrus. Scatterplots are presented only for illustrative purposes. 


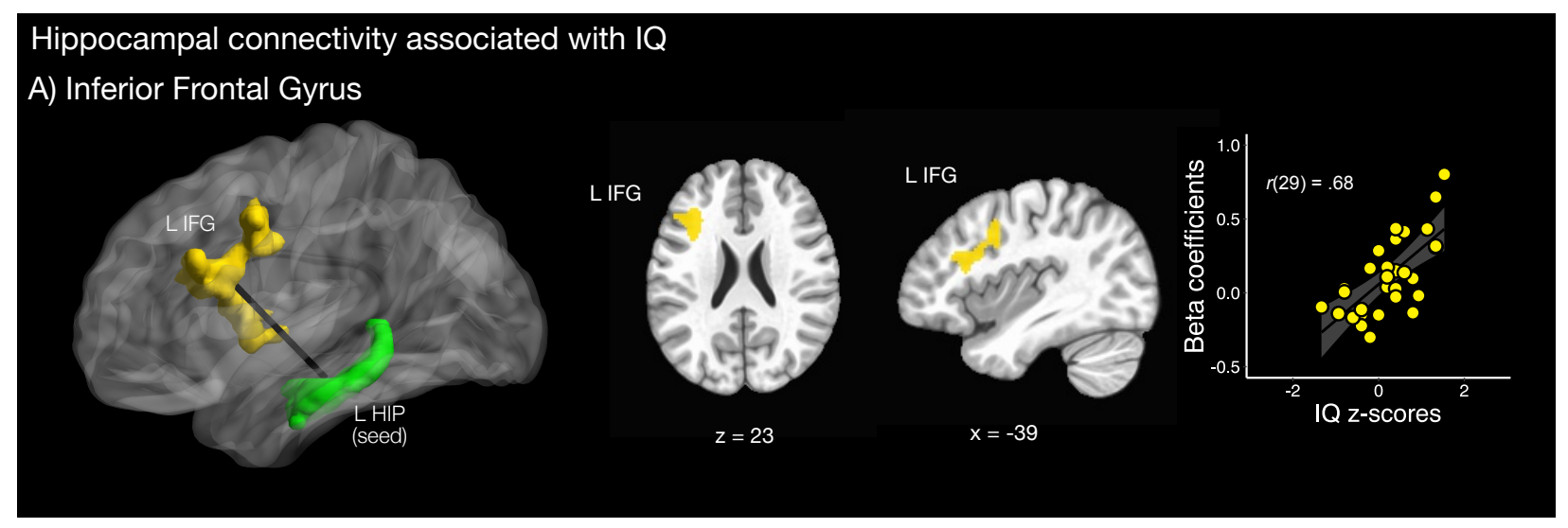

Figure 5. Left hippocampal functional connectivity associated with IQ. A) In contrast to math ability, IQ scores were positively related to the functional connectivity between the left hippocampus and a cluster in the inferior frontal gyrus (height threshold $p<.01$, extent threshold $p<.10)$. Note. $\mathrm{L}=$ left, $\mathrm{R}=$ right, $\mathrm{HIP}=$ hippocampus, $\mathrm{IFG}=$ inferior frontal gyrus. Scatterplots are presented only for illustrative purposes. 
Table 1. Descriptive statistics and correlations among age, WRAT Math Computation (raw score), and other standardized measures (standardized and scale scores)

\begin{tabular}{rccccrrrrr}
\hline Measure & M & SD & Range & 1 & 2 & 3 & 4 & 5 & 6 \\
\hline 1. Age $^{\mathrm{n}}$ & 16.42 & 0.8 & $14.97-17.83 \mathrm{y}$ & - & -0.11 & -0.24 & -0.04 & -0.05 & 0.01 \\
2. WISC IQ $^{\mathrm{a}}$ & 103.45 & 10.96 & $80-123$ & & - & $0.40^{*}$ & $0.72^{* * *}$ & $0.63^{* * *}$ & $0.70^{* * *}$ \\
3. ENI Reading a Text Aloud $^{\mathrm{b}}$ & 10.45 & 2.22 & $7-15$ & & & - & $0.39^{*}$ & 0.29 & $0.50^{* *}$ \\
4. WRAT Math Computation $^{\mathrm{c}}$ & 27.97 & 8.46 & $10-38$ & & & & - & $0.93^{* * *}$ & $0.54^{* *}$ \\
5. ENI Written Math & 13.58 & 6.28 & $2-19$ & & & & & - & 0.23 \\
6. WISC Arithmetic $^{\mathrm{b}}$ & 11.32 & 2.86 & $6-17$ & & & & & - \\
\hline
\end{tabular}

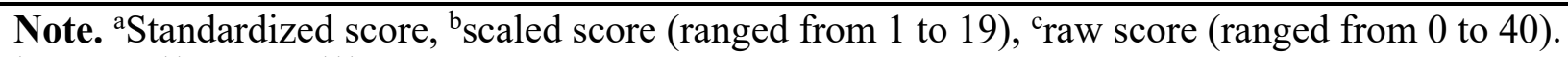
${ }^{*} p<.05,{ }^{* *} p<.01,{ }^{* * *} p<.001$ 
Table 2.

Left IPS functional connectivity associated with mathematical performance after controlling for

IQ.

\begin{tabular}{|c|c|c|c|c|c|}
\hline \multirow[b]{2}{*}{ Seed ROI } & \multirow[b]{2}{*}{ Cluster Size } & \multirow[b]{2}{*}{ Peak $(Z)$} & \multicolumn{3}{|c|}{ Coordinates } \\
\hline & & & $\mathrm{x}$ & $\mathrm{y}$ & $\mathrm{z}$ \\
\hline \multicolumn{6}{|l|}{ Left IPS } \\
\hline \multicolumn{6}{|l|}{ Negative correlation } \\
\hline Right lateral occipital cortex ${ }^{*}$ & 1641 & -4.03 & 29 & -79 & 3 \\
\hline Left fusiform gyrus ${ }^{\dagger}$ & 923 & -3.84 & -19 & -87 & -1 \\
\hline \multicolumn{6}{|l|}{ Positive correlation } \\
\hline \multicolumn{6}{|l|}{ N.S. } \\
\hline \multicolumn{6}{|l|}{ Right IPS } \\
\hline \multicolumn{6}{|l|}{ Negative correlation } \\
\hline \multicolumn{6}{|l|}{ N.S. } \\
\hline \multicolumn{6}{|l|}{ Positive correlation } \\
\hline N.S. & & & & & \\
\hline
\end{tabular}

Note. ${ }^{*}$ Extent threshold $p<.05,{ }^{\dagger}$ extent threshold $p<.10$ 


\section{Table 3.}

Hippocampal functional connectivity associated with mathematical performance after controlling for IQ

\begin{tabular}{|c|c|c|c|c|c|}
\hline \multirow[b]{2}{*}{ Seed ROI } & \multirow[b]{2}{*}{ Cluster Size } & \multirow[b]{2}{*}{ Peak $(Z)$} & \multicolumn{3}{|c|}{ Coordinates } \\
\hline & & & $\mathrm{x}$ & $\mathrm{y}$ & $\mathrm{z}$ \\
\hline \multicolumn{6}{|l|}{ Left hippocampus } \\
\hline \multicolumn{6}{|l|}{ Negative correlation } \\
\hline Left precentral gyrus ${ }^{\dagger}$ & 1001 & -4.64 & -41 & -17 & 65 \\
\hline Left insula (central opercular) ${ }^{\dagger}$ & 921 & -4.33 & -37 & 1 & 21 \\
\hline \multicolumn{6}{|l|}{ Positive correlation } \\
\hline \multicolumn{6}{|l|}{ N.S. } \\
\hline \multicolumn{6}{|l|}{ Right hippocampus } \\
\hline \multicolumn{6}{|l|}{ Negative correlation } \\
\hline \multicolumn{6}{|l|}{$N . S}$. \\
\hline \multicolumn{6}{|l|}{ Positive correlation } \\
\hline N.S. & & & & & \\
\hline
\end{tabular}

Note. ${ }^{\dagger}$ Extent threshold $p<.10$ 
Table 4.

Hippocampal functional connectivity associated with IQ.

\begin{tabular}{|c|c|c|c|c|c|}
\hline \multirow[b]{2}{*}{ Seed ROI } & \multirow[b]{2}{*}{ Cluster Size } & \multirow[b]{2}{*}{ Peak $(Z)$} & \multicolumn{3}{|c|}{ Coordinates } \\
\hline & & & $\mathrm{x}$ & $\mathrm{y}$ & $\mathrm{z}$ \\
\hline \multicolumn{6}{|l|}{ Left hippocampus } \\
\hline \multicolumn{6}{|l|}{ Negative correlation } \\
\hline \multicolumn{6}{|l|}{ N.S. } \\
\hline \multicolumn{6}{|l|}{ Positive correlation } \\
\hline Left inferior frontal gyrus $^{\dagger}$ & 998 & 4.15 & -35 & 17 & 23 \\
\hline \multicolumn{6}{|l|}{ Right hippocampus } \\
\hline \multicolumn{6}{|l|}{ Negative correlation } \\
\hline \multicolumn{6}{|l|}{$N . S$} \\
\hline \multicolumn{6}{|l|}{ Positive correlation } \\
\hline N.S. & & & & & \\
\hline
\end{tabular}

Note. 'Extent threshold $p<.10$ 